\title{
Targeted deep sequencing of mucinous ovarian tumors reveals multiple overlapping RAS-pathway activating mutations in borderline and cancerous neoplasms
}

Robertson Mackenzie', Stefan Kommoss ${ }^{2,3}$, Boris J. Winterhoff4, Benjamin R. Kipp ${ }^{4}$, Joaquin J. Garcia ${ }^{4}$, Jesse Voss ${ }^{4}$, Kevin Halling ${ }^{4}$, Anthony Karnezis ${ }^{2}$, Janine Senz ${ }^{2}$, Winnie Yang ${ }^{1}$, Elena-Sophie Prigge ${ }^{5}$, Miriam Reuschenbach ${ }^{5}$, Magnus Von Knebel Doeberitz ${ }^{5}$, Blake C. Gilks², David G. Huntsmann², Jamie Bakkum-Gamez ${ }^{7}$, Jessica N. McAlpine and Michael S. Anglesio $2,8^{*}$

\begin{abstract}
Background: Mucinous ovarian tumors represent a distinct histotype of epithelial ovarian cancer. The rarest (2-4 \% of ovarian carcinomas) of the five major histotypes, their genomic landscape remains poorly described. We undertook hotspot sequencing of 50 genes commonly mutated in human cancer across 69 mucinous ovarian tumors. Our goals were to establish the overall frequency of cancer-hotspot mutations across a large cohort, especially those tumors previously thought to be "RAS-pathway alteration negative", using highly-sensitive next-generation sequencing as well as further explore a small number of cases with apparent heterogeneity in RAS-pathway activating alterations.

Methods: Using the lon Torrent PGM platform, we performed next generation sequencing analysis using the v2 Cancer Hotspot Panel. Regions of disparate ERBB2-amplification status were sequenced independently for two mucinous carcinoma (MC) cases, previously established as showing ERBB2 amplification/overexpression heterogeneity, to assess the hypothesis of subclonal populations containing either KRAS mutation or ERBB2 amplification independently or simultaneously.
\end{abstract}

Results: We detected mutations in KRAS, TP53, CDKN2A, PIK3CA, PTEN, BRAF, FGFR2, STK11, CTNNB1, SRC, SMAD4, GNA11 and ERBB2. KRAS mutations remain the most frequently observed alteration among MC (64.9\%) and mucinous borderline tumors (MBOT) (92.3\%). TP53 mutation occurred more frequently in carcinomas than borderline tumors (56.8\% and $11.5 \%$, respectively), and combined IHC and mutation data suggest alterations occur in approximately $68 \%$ of MC and as many as $20 \%$ of MBOT. Proven and potential RAS-pathway activating changes were observed in all but one MC. Concurrent ERBB2 amplification and KRAS mutation were observed in a substantial number of cases (7/63 total), as was co-occurrence of KRAS and BRAF mutations (one case). Microdissection of ERBBZ-amplified regions of tumors harboring KRAS mutation suggests these alterations are occurring in the same cell populations, while consistency of KRAS allelic frequency in both ERBB2 amplified and non-amplified regions suggests this mutation occurred in advance of the amplification event.

(Continued on next page)

\footnotetext{
* Correspondence: manglesio@bccrc.ca

${ }^{2}$ Pathology and Laboratory Medicine, University of British Columbia,

Vancouver, Canada

${ }^{8}$ Department of Pathology and Laboratory Medicine, University of British

Columbia, Vancouver, Canada

Full list of author information is available at the end of the article
}

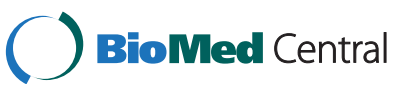

(c) 2015 Mackenzie et al.; licensee BioMed Central. This is an Open Access article distributed under the terms of the Creative Commons Attribution License (http://creativecommons.org/licenses/by/4.0), which permits unrestricted use, distribution, and reproduction in any medium, provided the original work is properly credited. The Creative Commons Public Domain Dedication waiver (http://creativecommons.org/publicdomain/zero/1.0/) applies to the data made available in this article, unless otherwise stated. 
(Continued from previous page)

Conclusions: Overall, the prevalence of RAS-alteration and striking co-occurrence of pathway "double-hits" supports a critical role for tumor progression in this ovarian malignancy. Given the spectrum of RAS-activating mutations, it is clear that targeting this pathway may be a viable therapeutic option for patients with recurrent or advanced stage mucinous ovarian carcinoma, however caution should be exercised in selecting one or more personalized therapeutics given the frequency of non-redundant RAS-activating alterations.

Keywords: Next-generation sequencing, Mucinous, Ovarian, BRAF, KRAS, TP53, Heterogeneity

\section{Background}

Mucinous ovarian tumors are a rare histological type of epithelial ovarian cancer (EOC), representing 2-4\% of these malignancies [1-4]. Primary mucinous ovarian carcinomas are distinct from other EOC in both presentation and outcome [3, 5-8]. Believed to develop along a continuum from benign cysts to borderline tumors to invasive carcinomas, the majority of cases present as borderline tumors (MBOT) or stage I mucinous carcinomas (MC). Overall, prognosis is excellent, although in rare cases where cancer has spread beyond the ovaries, outcomes and response to conventional chemotherapy is poor.

In addition to sharing many biomarkers, MCs are morphologically similar to adenocarcinomas of the pancreas and gastrointestinal tract, posing a challenge in differentiating primary ovarian tumors from metastatic disease [9-11]. Given the number of shared features between these disease entities, including a dominance of RAS-activating changes, there is a potential for similar therapeutic strategies and "umbrella" trials in women with advanced stage or recurrent disease [12, 13].

Among mucinous tumors, the most prevalent mutations occur in the mitogen activated protein kinase (MAPK) pathway, including KRAS mutations and ERBB2 amplification/ overexpression [13]. Historically, KRAS mutations have been observed in greater than $75 \%$ of mucinous ovarian tumors, although differentiation of MBOT from $\mathrm{MC}$ and exclusion of metastatic disease have not consistently been applied in studies of this disease type [14-16]. Copy number analyses have implicated loss of heterozygosity of chromosomal regions $9 p, 17 p$ and $21 q$ in the potential development of these tumors [17]. Additional mutations have been observed in BRAF, TP53, PTEN, PIK3CA and more recently CDKN2A and RNF43 [14, 18-20]. However, rarity of the disease has limited large-scale analyses of mutational frequency among mucinous ovarian tumors $[19,21]$. Furthermore, apparent intratumoral heterogeneity among mucinous tumors represents an interesting challenge for molecular profiling and potential personalized therapeutic strategies $[13,22]$.

Our group recently reported on the most frequently observed molecular alterations across mucinous tumors, observing KRAS mutations in $43.6 \% \mathrm{MCs}$ and $78.8 \%$
MBOTs and ERBB2 amplification/overexpression in $18.8 \%$ MCs and $6.2 \%$ MBOTs, the latter being assessed by immunohistochemistry, fluorescent- and chromogenic-in situ hybridization (IHC, FISH \& CISH) [13]. This analysis suggested tumors lacking ERBB2 or KRAS abnormalities tend to have poor prognosis, raising the question of whether an alternative mutation may be contributing to the pathology of this group [13]. In the current study, we applied targeted deep sequencing to the same cohort from our previous study [13], acquiring data for $37 \mathrm{MC}$ and 26 MBOT. Two primary goals were sought: first, to search for molecular alterations that may contribute to the pathogenesis of mucinous tumors without apparent RAS-activating alterations and second, to investigate heterogeneity observed in seemingly rare RAS-pathway "double-hit" cases discovered in our previous study [13]. An outline of our sequencing strategy and resultant data is given in Fig. 1.

\section{Methods}

\section{Sample cohort}

Collection of specimens for experimental analysis was performed by the OVCARE tumor bank and the Mayo Clinic, use of material was approved by the UBC-BCCA Research ethics board. All specimens underwent review of pathology reports (authors CBG, JNM) as well as single slide review of sampled material (author ANK and MSA) to confirm diagnosis and establish cellularity. Assessment of HPV infection was performed [23] to rule out the possibility of rare metastasis from the cervix presenting with mucinous histology in the ovary and all cases were negative. DNA was extracted from formalin fixed paraffin embedded (FFPE) tissue for sequencing analysis. Where noted, microdissection of potentially distinct cell populations was performed using ERBB2-IHC stained sections as a guide.

\section{Ion torrent sequencing}

Although we attempted to include the entire cohort described in our previous study [13], we were limited by availability and quality of material. DNA isolated from FFPE tissue was available for 89 mucinous tumors, including $30 \mathrm{MBOT}$ and $59 \mathrm{MC}$. Following quality control processing (described below), $37 \mathrm{MC}$ and $26 \mathrm{MBOT}$ 


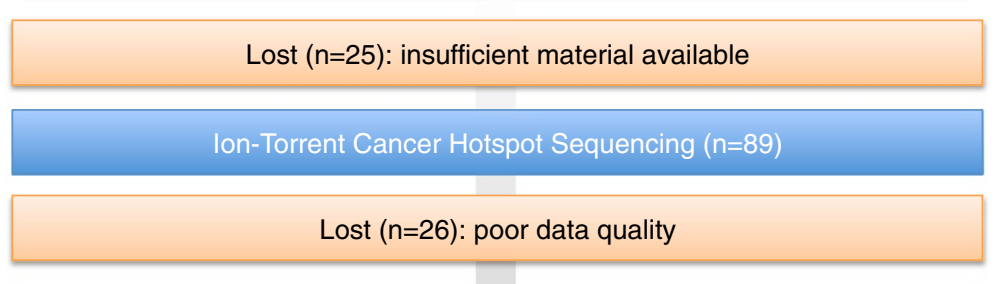

Cancer Hotspot Mutation Analysis $(n=63)$

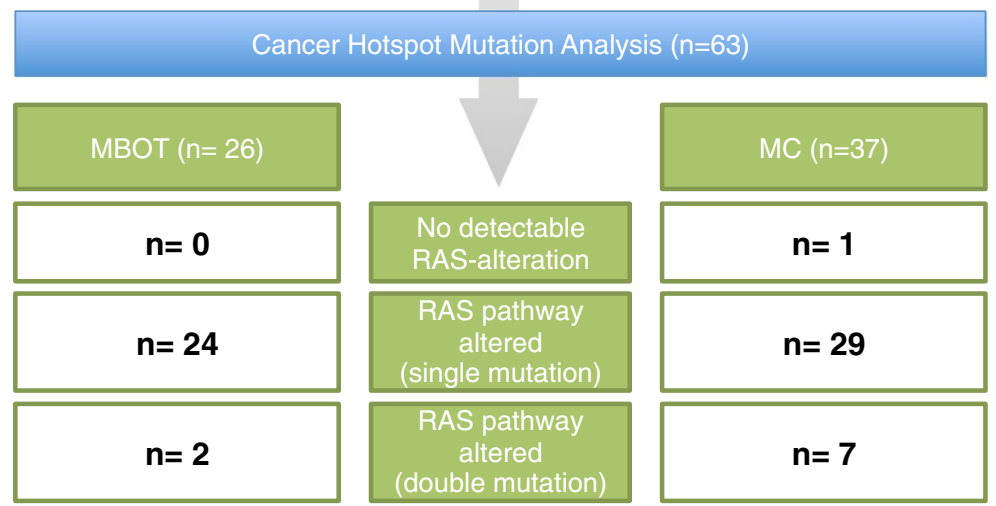

Fig. 1 Outline of next-generation sequencing based sequencing strategy in the context of previously established cohort RAS-alterations defined in Anglesio et al., 2013 [13]. Direct RAS-pathway alterations including suspected and known activating alteration to KRAS, BRAF, ERBB2, FGFR2, and STK11 (the latter is presumed to alleviate negative signals on mTOR via TSC1/2 complex, similar to the effect of ERK1/2 activation)

remained. Amplicon libraries were prepared and barcoded using the commercially available Cancer Hotspot Panel v2 primer pool and IonXpress barcode adapter kit as previously described [24, 25]. Libraries were quantified using Agilent High Sensitivity DNA chips, 20pM barcoded libraries were pooled (4 samples at a time), clonally amplified onto IonSphere particles using the Ion OneTouch system, and loaded on Ion 316 chips for sequencing. Variant calling was performed using the Ion Torrent Variant Caller with hg19 as a reference.

\section{Data processing and quality review}

Successful sequencing was defined when there was at least 100x average depth of coverage for $>80 \%$ of amplicons sequenced. Individual cases were manually reviewed to evaluate overall sequencing quality (e.g., the number of variant calls due to sequencing artifacts [26], percentage of reads mapping to target region, etc.). Cases with poor quality $(n=26)$ on manual review were excluded. We report only on variants observed at $>5 \%$ allelic frequency and $>10 \mathrm{x}$ coverage, that correspond to non-synonymous changes occurring in "hostspot" regions previously reported to be somatic in COSMIC (Catalogue of somatic mutations in cancer) [27], or are otherwise presumed to be deleterious and somatic if the given point mutation or insertion/deletion resulted in early termination.

\section{Immunohistochemistry}

ERBB2 IHC (scored according to ASCO/CAP guidelines [28]) was performed exactly as described in previously [13]. An ERBB2 IHC score of 3+ was used as a proxy for amplification status as this has been previously shown to be highly concordant in these and other tumor types (e.g. breast) $[13,29]$. IHC for p53 was generated as described previously [30] and scored on the same 3-tier system: $0=$ complete absence, $1=$ up to $50 \%$ nuclear positivity and $2=$ greater than $50 \%$ nuclear positivity. IHC for p53 was considered a proxy for mutations, where both the null phenotype (0) and strongly positive (2) were considered abnormal [30].

\section{Results}

\section{lon torrent sequencing}

Quality sequencing data was obtained for 63 cases of primary ovarian mucinous tumors including 26 borderline and 37 carcinomas (Fig. 1). Deleterious somatic mutations were observed within 13 genes: KRAS, TP53, CDKN2A, PIK3CA, PTEN, BRAF, FGFR2, STK11, CTNNB1, SRC, $S M A D 4$, GNA11, and ERBB2 (Table 1). Ion Torrent sequencing validated previously observed Sanger results for KRAS mutations [13] and identified three additional $K R A S$ variants that were not detectable by Sanger (likely due to low cellularity and restriction of the previous study to the amino acid 12/13 hotspot region. (Figs. 2 \& 3; 
Table 1 Somatic hotspot mutation frequencies for $M C$ and MBOT

\begin{tabular}{|c|c|c|}
\hline Carcinoma $(n=37)$ & Mutation Events & Frequency \\
\hline KRAS & 24 & 64.9 \\
\hline TP53 & 24 & 56.8 \\
\hline CDKN2A & 8 & 18.9 \\
\hline PIK3CA & 5 & 13.5 \\
\hline PTEN & 2 & 2.7 \\
\hline BRAF & 2 & 5.4 \\
\hline FGFR2 & 1 & 2.7 \\
\hline STK11 & 1 & 2.7 \\
\hline CTNNB1 & 2 & 5.4 \\
\hline$S R C$ & 1 & 2.7 \\
\hline SMAD4 & 1 & 2.7 \\
\hline Total Number Mutations & 71 & \\
\hline ERBB2 Amplification & 14 & 37.8 \\
\hline Borderline Tumor $(n=26)$ & Mutation Events & Frequency \\
\hline KRAS & 24 & 92.3 \\
\hline TP53 & 3 & 11.5 \\
\hline CDKN2A & 5 & 19.2 \\
\hline PIK3CA & 4 & 15.4 \\
\hline PTEN & 1 & 3.8 \\
\hline GNA11 & 1 & 3.8 \\
\hline ERBB2 & 1 & 3.8 \\
\hline Total Number Mutations & 39 & \\
\hline ERBB2 Amplification & $\S$ & 11.5 \\
\hline Total \# Mutations & 110 & \\
\hline
\end{tabular}

*Multiple cases with 2 mutation events. Number of mutated cases were used to establish frequency across cohort: TP53 $(n=21), \operatorname{CDKN} 2 A(n=7)$ and PTEN $(\mathrm{n}=1)$

${ }^{\S}$ Derived from Anglesio et al., 2013 [13]

Additional file 1). Additional variants were found in one MBOT and two MC: MBOT: VOA491 - p.Gly12Val; MC: OOU84 - p. Ala59Gly; and TMA3-41 - p.Gly12Val.

\section{Mucinous borderline tumors}

Among 26 MBOT cases, 39 presumed somatic mutations were detected across seven genes: KRAS (24/26; $92.3 \%)$, TP53 (3/26; $11.5 \%)$, CDKN2A (5/26; $19.2 \%)$, PIK3CA (4/ 26; $15.4 \%)$, PTEN (1/26; $3.8 \%)$, GNA11 (1/26; $3.8 \%)$, and ERBB2 (1/26; $3.8 \%$ ) (Table 1 \& Fig. 2). Amongst these MBOTs, KRAS mutations involved the "hotspot" for Gly12 only (Additional file 1). When grouped based on KRAS hotspot mutant and ERBB2 amplification status we observed 22 (84.6\%) KRAS+/ERBB2-, one (3.8\%) KRAS-/ ERBB2+, two (7.7 \%) KRAS+/ERBB2+, and one (3.8\%) KRAS-/ERBB2-; however, this last case harboured an ERBB2 p.Asp769Asn mutation rather than amplification. Despite the moderate frequency of amplification events, activating mutations of $E R B B 2$ have not previously been implicated in mucinous carcinoma pathogenesis. Mutations to the 769 residue are expected to have an activating effect given they are within the protein kinase domain [31-33]. Such mutations have been reported previously in both lung and esophageal cancers [34, 35].

\section{Mucinous carcinoma}

Within our cohort of $37 \mathrm{MC}$, we found 71 presumed somatic mutations within 11 different genes: KRAS (24/37; $64.9 \%)$, TP53 (21/37; $56.8 \%)$, CDKN2A (7/37; $18.9 \%)$, PIK3CA (5/37; 13.5 \%), PTEN (1/37; $2.7 \%)$, BRAF (2/ 37; $5.4 \%)$, FGFR2 (1/37; $2.7 \%)$, STK11 (1/37; $2.7 \%)$, CTNNB1 (2/37; 5.4 \%), SRC (1/37, $2.7 \%)$, and SMAD4 (1/37; $2.7 \%$ ) (Table 1 \& Fig. 3$)$. Three cases had two different, non-synonymous mutations in TP53 (OOU20, VOA439, TMA1-6), one case had two mutations observed in CDKN2A (OOU25), and one case had two PTEN mutations (TMA1-16). With a single exception (OOU84 p.Ala59Gly), KRAS mutations involved the Gly-12 residue. Co-occurrence of multiple mutations (including double hits to the RAS-pathway) was observed at a higher frequency within MC (26/37; $70.3 \%)$ over MBOT (12/26, $46.2 \%$ ), however was not statistically significant (Fisher exact test $\mathrm{p}=0.0634$ ).

Grouping of MC based on ERBB2 and KRAS status resulted in 19 (51.4 \%) KRAS+/ERBB2-, nine (24.3\%) $K R A S$-/ERBB2+, five (13.5 \%) KRAS+/ERBB2+, one (2.7\%) KRAS-/ERBB2-, and three KRAS- cases undefined ERBB2 amplification status (Fig. 3). Among the three KRAS-/ ERBB2 undefined cases, alternative RAS-pathway activating mutations were observed in two cases (TMA1-2: FGFR2 p.Ser252Trp; TMA3-12: BRAF p.Val600Glu), and the third (TMA2-39) having an STK11 inactivating change that may result in alleviation of negative signals on mTOR via TSC1/2 complex, similar to the effect of ERK1/2 activation [36, 37]. Ultimately, one case (TMA1-1) is definitively negative with respect to RAS-alteration status given the current screen.

\section{TP53 status amongst mucinous tumors}

Immunohistochemical scoring of p53 expression was generally concordant with mutation status (Figs. 2 \& 3). A TMA-based evaluation of p53 protein was done for the full cohort of Mayo and Vancouver samples, with interpretable results obtained for 15/26 MBOT and 29/37 $\mathrm{MC}$ where sequencing was also available. Of these, three MBOT cases had abnormal staining patterns for p53, and occurred in KRAS mutant or ERBB2 amplified cases. TMA1-23 and TMA3-49 showed complete loss of p53 staining; however, no mutation was observed in the regions sequenced, which may be the result of larger deletions or mutation outside of the hotspot panel. Twelve MC cases had abnormal p53 staining and appeared to be well distributed across all four groups of KRAS mutant 


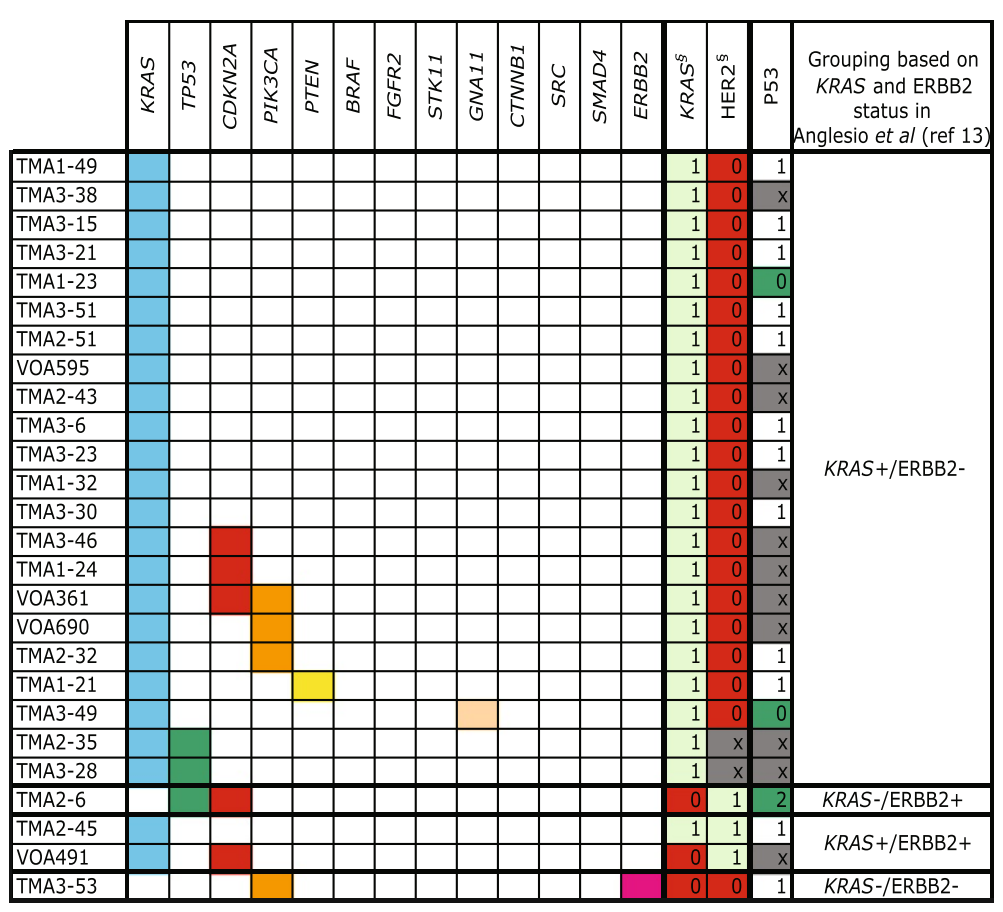

Fig. 2 Mutation frequencies and immunohistochemistry scores for 26 mucinous borderline tumors. Solid color in any of the first 13 columns represents a presumed somatic (COSMIC) hotspot mutation in the given case. In the last three columns numbers represent binarized IHC score for p53 and $\$$ "Original ERBB2 amplification and KRAS mutation" status derived from Anglesio et al., 2013 [13] where $0=$ Negative, $1=$ Positive, $X=$ Unknown, the latter derived from IHC, FISH, and/or CISH. IHC for p53 is displayed as three-tiered IHC score where 0 (no staining) and 2 ( $>50 \%$ positive nuclei) represent abnormal p53 status and 1 (1-50\% positive nuclei) represents normal p53 status ( $\mathrm{x}=$ data unavailable)

and ERBB2 amplified groups. Four cases (TMA3-31, OOU25, TMA1-36 and TMA1-1) had p53 staining abnormalities that occurred without detectable mutation. Finally, seven MC (18.9\%; TMA1-46, OOU 20, OOU 82, TMA2-16, TMA1-44, VOA 321 and VOA 695) were found to have presumed-somatic TP53 mutations, but did not have corresponding IHC abnormalities. It should be noted that the Ion-Torrent panel does not sequence the entirety of TP53 and is not well suited for the detection of exon-level (or larger) deletions, which may result in a null-phenotype by IHC. Further, our analysis may be partially confounded by non-somatic variants, whether contaminating the COSMIC database ("false-positive", non-somatic in our context), or having subtle effects on protein stability/unknown functional effects: i.e. the presence of a "presumed somatic mutation" may not yield a mutant overexpression or nullphenotype. Overall, TP53 mutations were more prevalent in MC, and no enrichment of TP53 was associated with any RAS-pathway mutation groups. Using p53 IHC data alone (Additional file 2) and expanding to all available cases, we observed no difference in overall or progression-free survival for the MC cohort (Additional file 3). Corresponding survival analysis for borderline tumors was uninformative due to cohort sample size and censoring. Our data set also failed to show enrichment of TP53 mutation, in either borderline or carcinomas.

\section{RAS-pathway heterogeneity}

Two cases of MC (VOA695 and VOA439) were previously described to be heterogeneous for ERBB2 amplification/ overexpression [13]. As greater access was available for these local cases, a full series of clinical blocks was examined for ERBB2 $3+$ and negative IHC. Positive and negative regions were then fine-needle microdissected with both front and back ERBB2-stained sections as a guide to ensure consistency in IHC positive $(3+)$ and negative $(0)$ regions. Sequencing of the disparate regions of VOA439 confirmed the previously observed KRAS p.Gly12Asp mutation at similar allelic frequency in both ERBB2+ and ERBB2- regions: $46.1 \%$ and $43.5 \%$ respectively (Fig. 4). Two TP53 and one CDKN2A mutations were also found in both regions at similar allelic frequencies. Similar results were observed in case VOA695 across ERBB2+ and ERBB2- regions: KRAS p.Gly12Asp mutation at $18.1 \%$ and $16.6 \%$, and TP53 p. Ser127Pro mutation at $10.5 \%$ and $19.6 \%$ allelic frequency, respectively. Doublehit RAS-pathway alterations were confirmed in six additional MC cases (total 21.6\%). Double-hits were observed in both MBOT (two cases; $7.7 \%$ ) and MC, but were more 


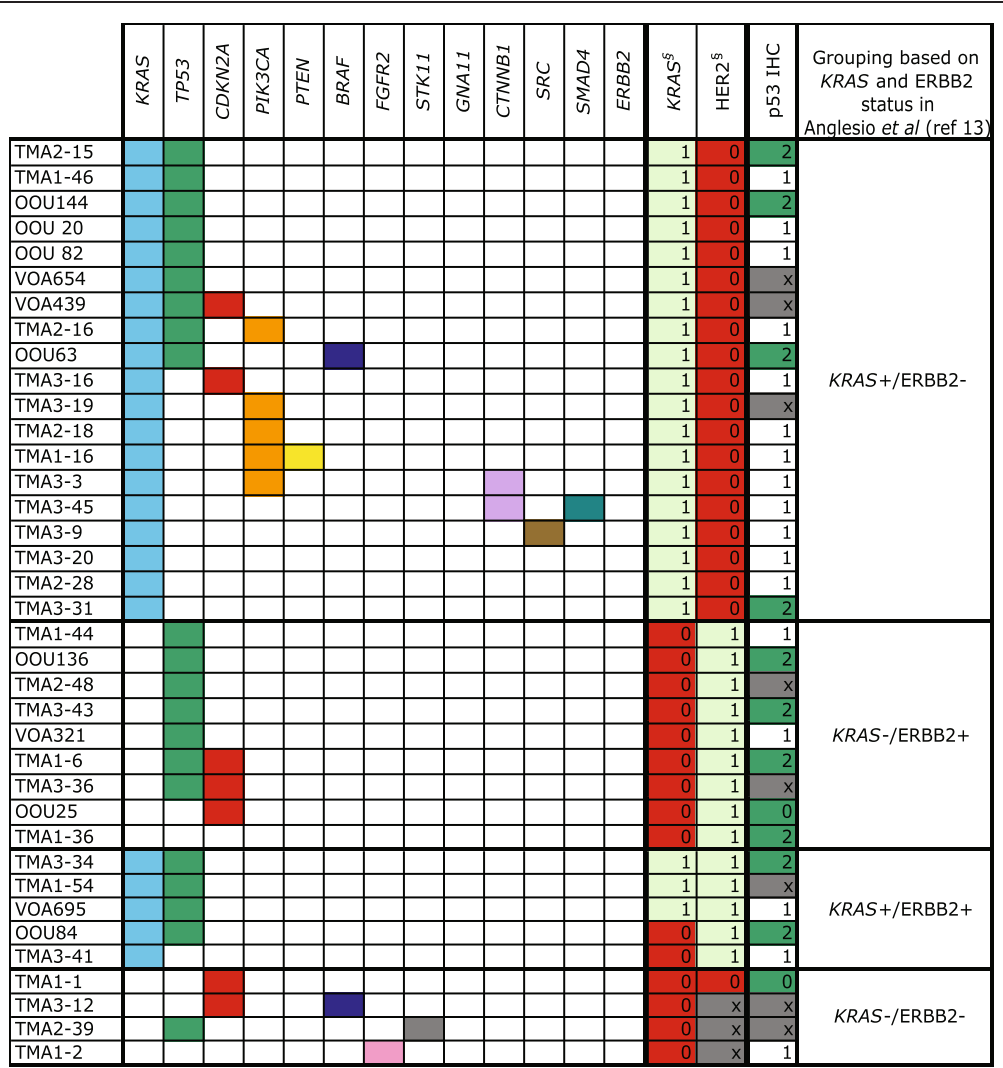

Fig. 3 Mutation frequencies and immunohistochemistry scores for 37 mucinous carcinoma. As in Fig. 2, Solid color in any of the first 13 columns represents a presumed somatic (COSMIC) hotspot mutation in the given case. In the last three columns numbers represent binarized IHC score for p53 and ${ }^{\S}$ "Original ERBB2 amplification and KRAS mutation" status derived from Anglesio et al., 2013 [13] where $0=$ Negative, $1=$ Positive, $\mathrm{X}=$ Unknown, the latter derived from $1 \mathrm{HC}, \mathrm{FISH}$, and/or CISH. IHC for p53 is displayed as three-tiered IHC score where 0 (no staining) and 2 (>50 \% positive nuclei) represent abnormal p53 status and 1 (1-50\% positive nuclei) represents normal p53 status

prevalent in MC. In general, examination of allelic ratios of RAS-pathway alterations in comparison to cellularity estimates suggested that RAS-pathway mutations may be more likely to be hemizygous or homozygous (Additional file 1) although copy number analysis was not available to validate this.

\section{Discussion}

In the current study we provide quantitative interrogation of MC and MBOTs using amplicon-based hotspot sequencing. Our re-sequencing efforts confirmed KRAS mutations to be the most frequent molecular alteration amongst mucinous tumors, appearing more common in borderline malignancies over carcinomas (92.3\% versus $64.9 \%$, respectively; Fisher exact $p=0.0157)$. These values reflect what was previously reported [13]; however, improved sensitivity through the use of next generation sequencing identified KRAS mutations in three cases previously believed to be wild type (one MBOT and two MC). We further added to the complement of known RAS-activating mutations in observing mutations in $B R A F$ (two $\mathrm{MC}$ ), as well as previously unreported potentially RAS-activating alterations in FGFR2, ERBB2, and STK11, each affecting a single carcinoma. As noted above, inactivating mutation of STK11 could be considered an alternative mechanism to RAS-activation outside of typical KRAS/BRAF mutations [36, 37], an important point given the occurrence of this mutation in one of only two MC without other known RAS alterations. Most other mutations observed here have previously been implicated in the biology of mucinous ovarian tumors (KRAS, BRAF, TP53, CDKN2A, PIK3CA, PTEN) $[14,15,18-20,38]$. Reported mutation frequencies vary, with small sample size and inconsistent diagnostic criteria likely at the heart of the variance observed in the literature. To the best of our knowledge, mutations within FGFR2, ERBB2 (missense/activating), STK11, GNA11, SRC, CTNNB1, and SMAD4 have not been previously reported in mucinous ovarian tumors. GNA11 mutations, such as the one observed in an MBOT have been shown to up-regulate RAS-pathway activation [39], and while $S R C$ mutations have not been previously reported in ovarian $\mathrm{MC}$, others have suggested a high level of SRC protein kinase activity in these tumors [40, 41]. 


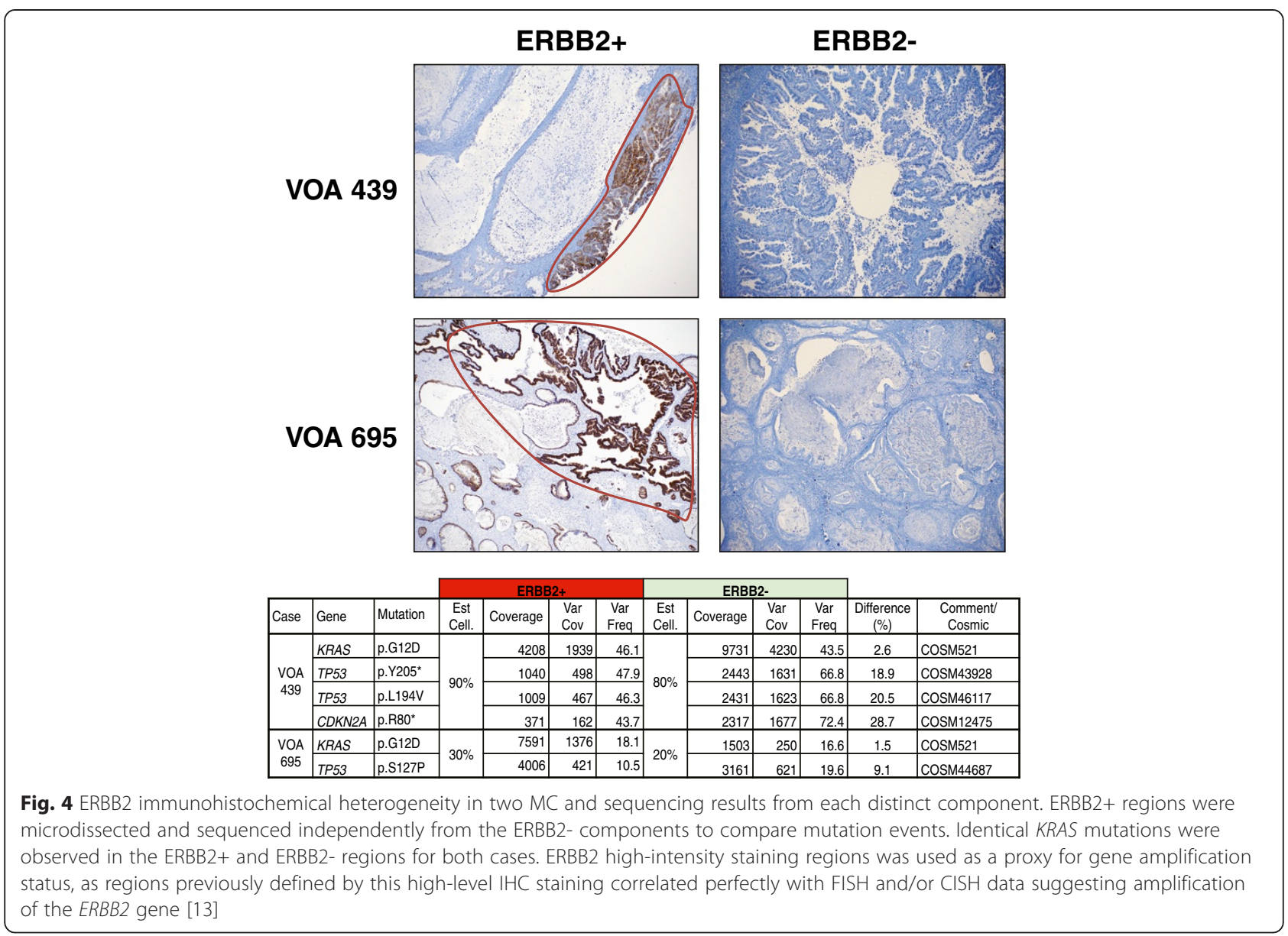

Amongst our cohort only one MC remained without identifiable RAS-pathway alteration, all but eradicating the RAS-activation negative group. Although relatively broad, our screen was not genome-wide and it is foreseeable that other rare RAS-activating alterations could be uncovered. This re-analysis also implies there is little difference in survival in tumors lacking RAS-pathway alterations, if any of these so-called "RAS-negative" tumors exist. We were also unable to show survival difference between ERBB2-positive, KRAS-positive, or non-

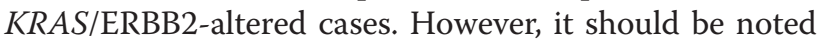
that our total cohort numbers have depleted since our previous analysis, and with additional RAS-pathway alterations defining unique groups, the number of samples per group were insufficient for meaningful conclusions on outcome.

Intratumoral heterogeneity among mucinous ovarian tumors, which previously seemed to be restricted to heterogeneity in ERBB2 status (observable in situ using FISH, CISH or IHC), presents a challenge for standard molecular analyses [13]. Based on our previous data suggesting a near-mutual exclusivity of RAS-pathway alterations in $\mathrm{MC}$ as well as numerous similar examples in the literature [42-44], we expected KRAS mutations would be restricted to regions lacking ERBB2 positivity. Surprisingly, KRAS mutations were found at near-identical frequencies in both ERBB2+ and ERBB2- regions of both examined MC. In fact, multiple alterations to the RASpathway were observed within two MBOT and six MC. This suggests that the KRAS mutations in both of these cases represent an ancestral alteration, present prior to the amplification of ERBB2. Further, this supports a model wherein RAS-pathway alterations are unlikely to be functionally equivalent.

Alterations involving the TP53 locus occurred more frequently in MC than MBOT (21/37; $56.8 \%$ and $3 / 26 ; 11.5 \%$, respectively). Aberrant expression of p53, assessed by IHC (scores of 0 and 2), suggest underlying genetic alterations in cases where no mutation were observed, a distinct possibility given the limits of our screening strategy. Considering both IHC and sequencing data, we estimate the frequency of TP53 alterations to be slightly higher than indicated in the mutation data alone and we estimate rates of approximately $20 \%$ and $68 \%$ for MBOT and $\mathrm{MC}$, respectively. Unfortunately, we were unable to show an effect for p53 mutation (based on IHC status or 
mutation status) on patient outcomes in either MBOT or MC (Additional file 3). It may be reasonable to suggest acquisition of TP53 mutation imparts genomic instability that in turn leads to accumulation of other mutations permissive overcoming senescence and other anti-growth signals induced by constitutive RAS-activation (for example through acquisition of PTEN loss of function mutations seen here). Should a suitable cohort be identified, a future study may be able to evaluate accumulated DNA copy number changes and clonal composition between MBOT and MUC. This may suggest a correlation between genomic complexity and acquisition of p53 mutations and/or secondary RAS-activating mutations, however this is conjecture at this point.

\section{Conclusions}

Previous data on mucinous ovarian cancers suggested a less favorable prognosis for cases not carrying a known RAS-pathway alteration [13], similar to reports in the ovarian low-grade serous/serous borderline tumor spectrum [45]. However, this finding is not reproducible in our current study where greater sensitivity in detection is applied and additional RAS-pathway alterations are considered. In general, we saw an increased frequency of multiple RAS-pathway alterations and TP53 mutations amongst carcinomas versus borderline tumors in our cohort, suggesting mutations in both of these pathways are critical in accelerating the progression of mucinous ovarian tumors. Save for a single case of MC, RAS-pathway activation is ubiquitious among mucinous ovarian tumors, in fact even this final case may have a cryptic RAS-activating alteration unseen by our hotspot screening strategy. Of particular importance, so-called "double hits" to this pathway were shown to overlap the same populations of cells in two cases where testing for this overlap was possible. This finding suggests different RAS mutations contribute, at least in part, unique functionality with respect to mucinous tumor progression.

Finally, the overall patterns of mutations amongst these tumors are not dissimilar to other mucinous tumor types, including pancreatic and appendiceal tumors [46-49]. Although extensive care was taken to exclude metastatic disease, limited certainty of primary ovarian tumor versus metastatic disease holds true for virtually all studies on $\mathrm{MC}$ and $\mathrm{MBOT}$, and remains a concern here. However, an overlapping relationship, either with respect to the origins or mechanisms mediating transformation, between ovarian mucinous and other peritoneal mucinous tumors is not unrealistic. Commonalities between these mucinous cancers may help explain the inherent chemoresistance in contrast to other EOC's and suggest so-called umbrella trial designs, grouping together cancers with similar molecular presentation, may provide a realistic option for treatment development in this relatively rare tumor type.

\section{Additional files}

Additional file 1: Hotspot sequencing, cellularity estimates and HPV infection status data for $\mathbf{2 6}$ mucinous borderline tumors and 37 mucinous carcinomas.

Additional file 2: p53 immunohistochemistry results and outcome data for entire cohort of MBOT and MC.

Additional file 3: Survival Analysis for MC based on p53

immunohistochemistry.

\section{Abbreviations}

$\mathrm{CISH}$ : Chromogenic-in situ hybridization; COSMIC: Catalogue of somatic mutations in cancer; EOC: Epithelial ovarian cancer; FFPE: Formalin fixed paraffin embedded; FISH: Fluorescent-in situ hybridization; IHC: Immunohistochemistry; MAPK: Mitogen activated protein kinase; MBOT: Mucinous borderline tumor; MC: Mucinous carcinoma.

\section{Competing interests}

The authors declare that they have no competing interests.

\section{Authors' contributions}

Samples used in this study were acquired by SK, BJW, JG, KH, JNM, and reviewed by ANK, JNM, DGH, and MSA with pathology oversight from CBG. Individual sample cellularity estimates were provided by ANK. HPV testing was performed by MVKD, ESP and MR on DNA extracted by JS, WY, SK, and RM. Panel sequencing was performed by RM, BRK, JV, and JS and data was analyzed by RM and BRK. JNM and MSA reviewed ERBB2 heterogeneity cases; tissue macrodissection and sequencing was performed by RTM. Immunohistochemical p53 staining and interpretation was provided by CBG and ANK. Study design and oversight was provided by $\mathrm{CBG}$, JNM and MSA. RM and MSA wrote the manuscript. All authors read and approved the final manuscript.

\section{Acknowledgements}

The Authors would like to thank the VGH and UBC Hospital Foundation and the BC Cancer Foundation, both of whom have contributed funding support to the Ovarian Cancer Research Team of BC (OVCARE; http://www.ovcare.ca). Funding bodies have no influence on research and the authors have no other relevant affiliations or financial involvement with any organization or entity with a financial interest in, or financial conflict with, the subject matter or materials discussed in the manuscript. No writing assistance was utilized in the production of this manuscript.

\section{Author details}

${ }^{1}$ Molecular Oncology, BC Cancer Agency Research Centre, Vancouver, Canada. ${ }^{2}$ Pathology and Laboratory Medicine, University of British Columbia, Vancouver, Canada. ${ }^{3}$ Gynecology and Obstetrics, Tuebingen University Hospital, Tuebingen, Germany. ${ }^{4}$ Laboratory Medicine and Pathology, Mayo Clinic, Rochester, USA. ${ }^{5}$ Applied Tumor Biology, Institute of Pathology, University of Heidelberg, Heidelberg, Germany. ${ }^{6}$ Gynecology and Obstetrics, Division of Gynecologic Oncology, University of British Columbia, Vancouver, Canada. ${ }^{7}$ Gynecology and Obstetrics, Mayo Clinic, Rochester, USA.

${ }^{8}$ Department of Pathology and Laboratory Medicine, University of British Columbia, Vancouver, Canada.

Received: 5 December 2014 Accepted: 6 May 2015 Published online: 19 May 2015

\section{References}

1. Gilks CB. Subclassification of ovarian surface epithelial tumors based on correlation of histologic and molecular pathologic data. Int J Gynecol Pathol. 2004;23(3):200-5.

2. Kurman RJ, Shih le M. Molecular pathogenesis and extraovarian origin of epithelial ovarian cancer-Shifting the paradigm. Hum Pathol. 201 1;42(7):918-31.

3. Tabrizi AD, Kalloger SE, Kobel M, Cipollone J, Roskelley CD, Mehl E, et al. Primary ovarian mucinous carcinoma of intestinal type: significance of pattern of invasion and immunohistochemical expression profile in a series of 31 cases. Int J Gynecol Pathol. 2010;29(2):99-107. 
4. Seidman JD, Horkayne-Szakaly I, Haiba M, Boice CR, Kurman RJ, Ronnett BM. The histologic type and stage distribution of ovarian carcinomas of surface epithelial origin. Int J Gynecol Pathol. 2004;23(1):41-4.

5. Alexandre J, Ray-Coquard I, Selle F, Floquet A, Cottu P, Weber B, et al. Mucinous advanced epithelial ovarian carcinoma: clinical presentation and sensitivity to platinum-paclitaxel-based chemotherapy, the GINECO experience. Ann Oncol. 2010;21(12):2377-81.

6. Pignata S, Ferrandina G, Scarfone G, Scollo P, Odicino F, Cormio G, et al. Activity of chemotherapy in mucinous ovarian cancer with a recurrence free interval of more than 6 months: results from the SOCRATES retrospective study. BMC Cancer. 2008:8:252.

7. Winter 3rd WE, Maxwell GL, Tian C, Carlson JW, Ozols RF, Rose PG, et al. Prognostic factors for stage III epithelial ovarian cancer: a Gynecologic Oncology Group Study. J Clin Oncol. 2007;25(24):3621-7.

8. Mackay HJ, Brady MF, Oza AM, Reuss A, Pujade-Lauraine E, Swart AM, et al. Prognostic relevance of uncommon ovarian histology in women with stage III/IV epithelial ovarian cancer. Int J Gynecol Cancer. 2010;20(6):945-52.

9. Seidman JD, Kurman RJ, Ronnett BM. Primary and metastatic mucinous adenocarcinomas in the ovaries: incidence in routine practice with a new approach to improve intraoperative diagnosis. Am J Surg Pathol. 2003;27(7):985-93.

10. Vang R, Gown AM, Wu LS, Barry TS, Wheeler DT, Yemelyanova A, et al. Immunohistochemical expression of CDX2 in primary ovarian mucinous tumors and metastatic mucinous carcinomas involving the ovary: comparison with CK20 and correlation with coordinate expression of CK7. Mod Pathol. 2006;19(11):1421-8.

11. Yemelyanova AV, Vang R, Judson K, Wu LS, Ronnett BM. Distinction of primary and metastatic mucinous tumors involving the ovary: analysis of size and laterality data by primary site with reevaluation of an algorithm for tumor classification. Am J Surg Pathol. 2008;32(1):128-38.

12. MCAlpine JN, Wiegand KC, Vang R, Ronnett BM, Adamiak A, Kobel M, et al. HER2 overexpression and amplification is present in a subset of ovarian mucinous carcinomas and can be targeted with trastuzumab therapy. BMC Cancer. 2009;9:433

13. Anglesio MS, Kommoss S, Tolcher MC, Clarke B, Galletta L, Porter H, et al. Molecular characterization of mucinous ovarian tumours supports a stratified treatment approach with HER2 targeting in $19 \%$ of carcinomas. J Pathol. 2013;229(1):111-20.

14. Cuatrecasas M, Villanueva A, Matias-Guiu X, Prat J. K-ras mutations in mucinous ovarian tumors: a clinicopathologic and molecular study of 95 cases. Cancer. 1997;79(8):1581-6.

15. Gemignani ML, Schlaerth AC, Bogomolniy F, Barakat RR, Lin O, Soslow R, et al. Role of KRAS and BRAF gene mutations in mucinous ovarian carcinoma. Gynecol Oncol. 2003;90(2):378-81.

16. Gurung A, Hung T, Morin J, Gilks CB. Molecular abnormalities in ovarian carcinoma: clinical, morphological and therapeutic correlates. Histopathology. 2013;62(1):59-70.

17. Feltmate CM, Lee KR, Johnson M, Schorge JO, Wong KK, Hao K, et al. Whole-genome allelotyping identified distinct loss-of-heterozygosity patterns in mucinous ovarian and appendiceal carcinomas. Clin Cancer Res. 2005:11(21):7651-7.

18. Hunter SM, Gorringe KL, Christie M, Rowley SM, Bowtell DD, Australian Ovarian Cancer Study G, et al. Pre-invasive ovarian mucinous tumors are characterized by CDKN2A and RAS pathway aberrations. Clin Cancer Res. 2012:18(19):5267-77.

19. Ryland GL, Hunter SM, Doyle MA, Rowley SM, Christie M, Allan PE, et al. RNF43 is a tumour suppressor gene mutated in mucinous tumours of the ovary. J Pathol. 2013;229(3):469-76.

20. Wang Y, Helland A, Holm R, Kristensen GB, Borresen-Dale AL. PIK3CA mutations in advanced ovarian carcinomas. Hum Mutat. 2005;25(3):322.

21. Rechsteiner M, Zimmermann AK, Wild PJ, Caduff R, von Teichman A, Fink D, et al. TP53 mutations are common in all subtypes of epithelial ovarian cancer and occur concomitantly with KRAS mutations in the mucinous type. Exp Mol Pathol. 2013;95(2):235-41.

22. Rubin SC, Finstad CL, Federici MG, Scheiner L, Lloyd KO, Hoskins WJ. Prevalence and significance of HER-2/neu expression in early epithelial ovarian cancer. Cancer. 1994;73(5):1456-9.

23. Reuschenbach M, Roos J, Panayotopoulos D, Baldus SE, Schnurch HG, Berger A, et al. Characterization of squamous cell cancers of the vulvar anterior fourchette by human papillomavirus, p16INK4a, and p53. J Low Genit Tract Dis. 2013;17(3):289-97.
24. Singh RR, Patel KP, Routbort MJ, Reddy NG, Barkoh BA, Handal B, et al. Clinical validation of a next-generation sequencing screen for mutational hotspots in 46 cancer-related genes. J Mol Diagn. 2013;15(5):607-22.

25. Lee CH, Hoang LN, Yip S, Reyes C, Marino-Enriquez A, Eilers G, et al. Frequent expression of KIT in endometrial stromal sarcoma with YWHAE genetic rearrangement. Modern Pathol. 2014;27(5):751-7.

26. Do H, Dobrovic A. Sequence artifacts in DNA from formalin-fixed tissues: causes and strategies for minimization. Clin Chem. 2015;61(1):64-71.

27. Forbes SA, Bindal N, Bamford S, Cole C, Kok CY, Beare D, et al. COSMIC: mining complete cancer genomes in the catalogue of somatic mutations in cancer. Nucleic Acids Res. 2011;39(Database issue):D945-50.

28. Wolff AC, Hammond ME, Schwartz JN, Hagerty KL, Allred DC, Cote RJ, et al. American Society of Clinical Oncology/College of American Pathologists guideline recommendations for human epidermal growth factor receptor 2 testing in breast cancer. J Clin Oncol. 2007;25(1):118-45.

29. Dowsett M, Bartlett J, Ellis IO, Salter J, Hills M, Mallon E, et al. Correlation between immunohistochemistry (HercepTest) and fluorescence in situ hybridization (FISH) for HER-2 in 426 breast carcinomas from 37 centres. J Pathol. 2003:199(4):418-23.

30. Kalloger SE, Kobel M, Leung S, Mehl E, Gao D, Marcon KM, et al. Calculator for ovarian carcinoma subtype prediction. Mod Pathol. 2011;24(4):512-21.

31. Coussens L, Yang-Feng TL, Liao YC, Chen E, Gray A, McGrath J, et al. Tyrosine kinase receptor with extensive homology to EGF receptor shares chromosomal location with neu oncogene. Science. 1985;230(4730):1132-9.

32. Lee JW, Soung YH, Seo SH, Kim SY, Park CH, Wang YP, et al. Somatic mutations of ERBB2 kinase domain in gastric, colorectal, and breast carcinomas. Clin Cancer Res. 2006;12(1):57-61.

33. Yamamoto T, Ikawa S, Akiyama T, Semba K, Nomura N, Miyajima N, et al. Similarity of protein encoded by the human c-erb-B-2 gene to epidermal growth factor receptor. Nature. 1986;319(6050):230-4.

34. Cancer Genome Atlas Research N. Comprehensive genomic characterization of squamous cell lung cancers. Nature. 2012;489(7417):519-25.

35. Dulak AM, Stojanov P, Peng S, Lawrence MS, Fox C, Stewart C, et al. Exome and whole-genome sequencing of esophageal adenocarcinoma identifies recurrent driver events and mutational complexity. Nat Genet. 2013:45(5):478-86.

36. Green AS, Chapuis N, Maciel TT, Willems L, Lambert M, Arnoult C, et al. The LKB1/AMPK signaling pathway has tumor suppressor activity in acute myeloid leukemia through the repression of mTOR-dependent oncogenic mRNA translation. Blood. 2010;116(20):4262-73.

37. Wang J, Whiteman MW, Lian H, Wang G, Singh A, Huang D, et al. A non-canonical MEK/ERK signaling pathway regulates autophagy via regulating Beclin 1. J Biol Chem. 2009;284(32):21412-24.

38. Saito M, Okamoto A, Kohno T, Takakura S, Shinozaki H, Isonishi S, et al. Allelic imbalance and mutations of the PTEN gene in ovarian cancer. Int J Cancer. 2000;85(2):160-5.

39. Gessi M, Hammes J, Lauriola L, Dorner E, Kirfel J, Kristiansen G, et al. GNA11 and N-RAS mutations: alternatives for MAPK pathway activating GNAQ mutations in primary melanocytic tumours of the central nervous system. Neuropathol Appl Neurobiol. 2013;39(4):417-25.

40. Liu T, Hu W, Dalton HJ, Choi HJ, Huang J, Kang Y, et al. Targeting SRC and tubulin in mucinous ovarian carcinoma. Clin Cancer Res. 2013;19(23):6532-43.

41. Matsuo K, Nishimura M, Bottsford-Miller JN, Huang J, Komurov K, Armaiz-Pena GN, et al. Targeting SRC in mucinous ovarian carcinoma. Clin Cancer Res. 2011;17(16):5367-78.

42. Davies H, Bignell GR, Cox C, Stephens P, Edkins S, Clegg S, et al. Mutations of the BRAF gene in human cancer. Nature. 2002:417(6892):949-54.

43. Rajagopalan H, Bardelli A, Lengauer C, Kinzler KW, Vogelstein B, Velculescu VE. Tumorigenesis: RAF/RAS oncogenes and mismatch-repair status. Nature 2002;418(6901):934.

44. Anglesio MS, Arnold JM, George J, Tinker AV, Tothill R, Waddell N, et al. Mutation of ERBB2 provides a novel alternative mechanism for the ubiquitous activation of RAS-MAPK in ovarian serous low malignant potential tumors. Mol Cancer Res. 2008;6(11):1678-90.

45. Grisham RN, lyer G, Garg K, DeLair D, Hyman DM, Zhou Q, et al. BRAF mutation is associated with early stage disease and improved outcome in patients with low-grade serous ovarian cancer. Cancer. 2013;119(3):548-54.

46. Abraham SC, Klimstra DS, Wilentz RE, Yeo CJ, Conlon K, Brennan M, et al. Solid-pseudopapillary tumors of the pancreas are genetically distinct from pancreatic ductal adenocarcinomas and almost always harbor beta-catenin mutations. Am J Pathol. 2002;160(4):1361-9. 
47. Hidalgo M. Pancreatic cancer. N Engl J Med. 2010;362(17):1605-17.

48. Tanaka Y, Kato K, Notohara K, Hojo H, ljiri R, Miyake T, et al. Frequent beta-catenin mutation and cytoplasmic/nuclear accumulation in pancreatic solid-pseudopapillary neoplasm. Cancer Res. 2001;61(23):8401-4.

49. Liu X, Mody K, de Abreu FB, Pipas JM, Peterson JD, Gallagher TL, et al. Molecular profiling of appendiceal epithelial tumors using massively parallel sequencing to identify somatic mutations. Clin Chem. 2014;60(7):1004-11.

Submit your next manuscript to BioMed Central and take full advantage of:

- Convenient online submission

- Thorough peer review

- No space constraints or color figure charges

- Immediate publication on acceptance

- Inclusion in PubMed, CAS, Scopus and Google Scholar

- Research which is freely available for redistribution 